\title{
Social isolation and loneliness in old age: Exploring their role in mental and physical health
}

\author{
Panagiota Tragantzopoulou, Vaitsa Giannouli
}

School of Psychology, Mediterranean College - University of Derby, Thessaloniki, Greece

ARTICLE HISTORY: Received 9 July 2020/Revised 21 September 2020/Published Online 17 March 2021

\begin{abstract}
The current review has the ultimate scope to accurately define social isolation and loneliness while highlighting the serious repercussions on health and behavior. Daily hundreds of people across the globe report suffering from social isolation and loneliness; an overwhelming feeling of emptiness, unworthiness and personal failure. Human beings are social species that have the need to nurture reliable and secure social settings to survive. Simultaneously, trustworthy social relationships are critical for mental and physical wellbeing whereas impaired social interactions can lead to social isolation and loneliness. In an attempt to tease out and elucidate salient problems and issues, we seek to critically compose studies, views and issues from a variety of perspectives by providing opposing standpoints and conversational voices instead of intensifying traditional narratives and dominant discourses. Few aspects of social isolation and loneliness are untouched by scientific attention. The role of these concepts in old age is no exception and arguably has a tremendous impact in multiple aspects of life. Social isolation and loneliness are two distinctive concepts that have been identified as risk factors for wellbeing, health and everyday functioning in profound ways. Therefore, we aimed to examine the associations with various psychiatric disorders for instance anxiety, depression, psychotic disorder and Alzheimer's disease as well as with a variety of physical disorders such as cardiovascular diseases, cholesterol and autoimmune diseases. Given the alarming records from healthcare which depict an almost two-fold rise in healthcare attendance, we attempt to assemble the proposed interventions through an in-depth review of the current literature available and provide the incentive for constructive and collective thought. To our theoretical understanding, in order to better comprehend these psychosocial concepts and deliver timely and more effective personalized interventions to those in need, it is of paramount importance to thoroughly examine the identified causal links. However, further research is required for the reduction or the deletion of the undesired effects.
\end{abstract}

KEYWORDS: Social isolation, emotional loneliness, Alzheimer's disease, psychosis, interventions.

\section{Social isolation and loneliness:}

\section{Definitions, gender and cultural differences}

It is, sometimes, very intricate to discern social isolation from loneliness since not all isolated individuals are lonely and not all who feel lonely are secluded. Social isolation is considered an objective, quantitative parameter that indicates the size of the individual's network and the frequency of contact. ${ }^{1}$ Following definitions and approaches in sociology and psychol- ogy, isolation is segregated in two forms, objective and subjective isolation. Objective isolation can be described by situational factors for example lack or infrequent contact with network members and absence of participation in activities. ${ }^{2}$ On the other side, subjective isolation can be determined by subjective factors such as shortfall of social network diversity and resources of partnership. ${ }^{3}$ Similarly, when defining loneliness, most of the researchers emphasize on the actual or perceived deficits that social relation- 
ships project. It represents a qualitative, subjective parameter referencing the individual's expectancy and gratification with the periodicity and proximity of contacts. ${ }^{4}$ Loneliness is conceptualized as emotional and social loneliness. Emotional loneliness represents the perceived lack of meaningful attachment whereas social loneliness represents the lack of membership to a group. ${ }^{5}$

It is hardly surprising that feelings of loneliness are associated with social isolation, albeit existing studies concluded that the association is weak to moderate. ${ }^{6}$ Cornwell \& Waite ${ }^{7}$ suggest that the relationship between loneliness and social isolation in older adults may be 'decoupled' as a consequence of their expectation for, and as an extension of their preparation, transitions and declines in their social network. Evidence from twin studies reveal that loneliness can have a heritable cause and maintain a stable course, with findings attributing almost equal percentages to heritage and non-shared environmental factors in adulthood. ${ }^{8}$ However, concrete situational factors augment the risk for experiencing loneliness. Based on several studies, these factors include deficiency in social relations, few social roles, incapacity to be vigorously involved in the local community activities, low socioeconomic status, physical health symptoms and poor marital status. ${ }^{9,10}$ In the transitional process from middle age to older age, social roles are being disrupted and several alterations in both the individual and the family level supervene. Initially, this chronological stage is marked by their release from the labor force and their integration to the retired population. Accordingly, individuals experience the parenthood to 'empty nest' phase which tangles the possible attenuation of kinship attachments. ${ }^{11}$ Literature reviews conclude that the empty-nest syndrome is a transitional period that can be characterized by negative effects such as depression, alcoholism, identity crisis, marital conflict and isolation. ${ }^{12}$ Equally, they face the inevitable emotional pain resulting from the death of their loved ones and the loss of family and friendship ties. ${ }^{13}$

Gender differences have been identified depicting a higher prevalence in the female population. Researchers believe that although females are at risk of more ill-health conditions, they tend to outlive their partners. ${ }^{14}$ Studies have also shown that women are widely integrated in social networks whereas men are to lesser degree recipients of social support. ${ }^{15}$ Accordingly, an important aspect that should be mentioned is that women tend to have more social support exchanges and maintain more kinship ties and family connections compared to men, perhaps because childlessness is highly valued. ${ }^{16}$ Cultural and cross-national differences were also evident. Tomaka et $\mathrm{al}^{17}$ attempted to make comparisons between Caucasian and Hispanic samples. Based on their research, Hispanics are more prone to experience loneliness and develop health-related problems such as diabetes and hypertension. They, also, stated that having a sense of belonging somewhere and having a source of support could be protective and eliminate the risk of these illnesses. Hence, it is of fundamental importance to examine loneliness within specific cultures given that mental health can be acutely affected by culture.

\section{Social isolation and loneliness: Relations to mental health}

The experience of feeling isolated and lonely can have detrimental effects on mental and physical wellbeing while it has been scientifically related to a heightened danger of unfolding serious health issues. High levels of loneliness and social isolation have been observed in individuals with mental health conditions, hearing and vision impairments, and chronic health problems and in neurodivergent groups such as autistic individuals.

\section{Anxiety}

Studies have reported links between loneliness and social anxiety in older adults. As stated by Fry and Debats some elderly people with self-expectancies or internalized beliefs about their aging can experience severe anxiety connected with feelings of loneliness. ${ }^{18}$ A longitudinal study with more than 1,100 adult participants which measured loneliness in a period of six months revealed that early state loneliness could predict later state social anxiety, paranoia and depression. ${ }^{19}$ Further, a high-performance electroencephalographic (EEG) study that examined social stimuli in lonely and non- lonely older individuals supported the evolutionary theory of loneliness which denotes that lonely individuals are constantly alarmed and display heightened attention for social threat during the early stages of processing social stimuli, by reporting differences in the process of social and non- social threat stimuli in the first $116 \mathrm{~ms}$ of information processing. ${ }^{20,21}$ Despite the irrefutable evidence associating loneliness with social anxiety among several age groups, links to other forms of anxiety remain largely underexplored.

\section{Depression}

When referring to social isolation and loneliness in older populations, it is vital to take into account depression as another strong confounding factor that can 
provoke serious behavioral and biological implications. According to $\mathrm{WHO}$, depression among elderly has a prevalence of $10-20 \% .{ }^{22}$ As ubiquitous life-changing events and physical disablement evolve, demoralization and depression commonly accompany them. There are cases that overt depressive symptomatology for instance social withdrawal, distress, idleness and melancholy veil the widespread loneliness. Empirical studies determined that loneliness and depression are distinctive concepts both statistically and functionally, ${ }^{23,24}$ with loneliness serving as a predicting factor for depression but not vice versa. ${ }^{25}$ Previous research has confirmed that loneliness and social isolation are considerably associated with depression and operate as a pathway through which individual's health is being affected with recent meta-analyses concluding that loneliness has moderately significant effect on depression. ${ }^{26}$ In fact, poorer health- related behaviors, risk for cardiovascular disease and higher levels of inflammatory markers are more likely to be reported by depressed individuals. ${ }^{27,28}$

Several studies suggest insecure attachment styles escalate susceptibility to social isolation, loneliness and depression. The susceptibility to depression can be caused due to the fact that insecurely attached individuals tend to have poor problem solving skills, an unstable self-concept, low self-esteem and difficulty in developing and maintaining relationships. ${ }^{29} \mathrm{~A}$ mixed method study of chronic depression in older British Pakistani women found that the persistence of depression was partly explained by social isolation..$^{30}$ Interestingly, Wilby found that depressed older people were not socially isolated but were on the contrary more likely to report contacts than non-depressed respondents. ${ }^{31}$ The discrepancy in findings could be better understood if the quality and meaning of different types of social relations in old age were explored. Although evidence about the association of loneliness and depression are well-established, several limitations were identified in the existing longitudinal studies. Firstly, there are other individual traits including objective social isolation, objective and perceived stress, and low social support that can be associated with loneliness and depression. However, it is not clear the extent to which each of these characteristics can affect depressive symptoms. Secondly, most prior studies relied on convenience samples without taking into consideration the potential implications of gender, ethnicity, education, psychiatric diagnosis, use of antidepressant medications, or physical functioning. Finally, their analyses relied on regression or latent growth models in which depressive symptomatology served as the criterion measure. ${ }^{25}$

\section{Alzheimer's disease}

Across the years, sparse studies have attempted to connect social isolation and loneliness with the onset of Alzheimer's disease (AD), but this relationship in people with $A D$ is relatively unknown. Previous research that looked into the relationship of social isolation and dementia has highlighted that quality is better than quantity. Amieva et al found that social interactions that provide the feelings of satisfaction and perceived reciprocity can serve as protective factors of dementia over 15 years whereas the size and nature of social networks was not associated with dementia risk. ${ }^{32}$ Further, a 12-year cohort study confirmed that having a confidant can secure individuals from dementia symptomatology. ${ }^{33}$ Other studies that explored the impact of loneliness on dementia onset found that older adults who felt lonely were more than twice as likely to develop an AD-like dementia syndrome, than those who were not lonely. ${ }^{34}$ Holwerda et al found that healthy older adults were more likely to develop clinical dementia after 3 years if they felt lonely, rather than being lonely at baseline. Research exploring loneliness in people who already have dementia is rare, probably because it is difficult to ascertain whether someone with cognitive problems can accurately evaluate how lonely he is feeling. ${ }^{35} \mathrm{Haj}$ et al found that participants with $A D$ were significantly lonelier than healthy controls, and this positively correlated with emotional deprivation. ${ }^{36}$

\section{Psychotic disorders}

Independently of genetic or biological factors, evidence reveals that social isolation and loneliness are risk factors for developing psychosis. Interestingly, EI Haj et al found in a sample of lonely participants that individuals with $A D$ and healthy elderly controls presented a greater incidence of hallucinations. ${ }^{36}$ This finding could possibly indicate that the onset of psychotic symptoms may be determined by loneliness even if underlying neurological or psychiatric conditions are absent. In accordance, studies have suggested that social isolation makes older individuals more susceptible to aberrant sensations across exteroceptive and proprioceptive dimensions, especially when the individual experiences self-disturbances and anomalous bodily experiences. ${ }^{37}$ Quadt et al highlight interoception meaning the body-to-brain communication through distinct neural and humoral channels that can result in impaired cognitive and emotional processes when the external and internal needs are not met and social isolation and loneliness serve as social allostatic overloads. ${ }^{21}$ While the effects of social isolation and loneli- 
ness on depressive symptomatology and $A D$ are widely recognized, the links among psychotic disorder, social isolation and loneliness remain vague and largely underexplored.

\section{Suicidal ideation}

High levels of mortality have been observed in both social isolation and loneliness and it is significantly possible that suicidal behavior can play a vital role for these high scores. Suicidal behavior is a complex biopsychosocial process that is considered a world- wide epidemic with almost 800.000 people worldwide dying each year by suicide. ${ }^{38}$ Research investigating the links among social isolation, loneliness and suicidal behavior is scarce with existing findings indicating that adolescents and elderly are vulnerable to both loneliness and suicidal behavior. ${ }^{39}$ Lonely individuals are prone to perceiving life as stressful and unbearable, with chronic stress being also related with high levels of suicidal ideation..$^{40} \mathrm{~A}$ recent narrative meta- analysis found that having no partner, living alone, social isolated, feeling lonely, feeling alienated from others, and feeling not to belong are the main social constructs that can highly contribute to suicidal outcomes. ${ }^{41}$ In this particular study, subjective loneliness had the greatest impact on both suicidal ideations and suicidal attempts. This outcome was reinforced by an integrative meta- analysis showing that the function and the quality of social relationships are more predictive of suicidal behavior. ${ }^{42}$ In view of these findings, meaningful social relationships seemingly can constitute a protective factor against suicidal behavior.

\section{Physical health}

The detrimental effects of social isolation and loneliness on physical health in old age are well-documented, with recent meta-analyses reporting a 30\% increased levels of poorer quality of sleep, stress, and risk for stroke and myocardial infarction. ${ }^{43}$ Academic literature has confirmed that these effects on individual's mortality are nearly identical to prime well-known health dangers for example smoking and alcohol consumption and surpass that of physical inaction and obesity. ${ }^{43}$ Nonetheless, research focusing solely on loneliness and health-risk behaviors reported either no significant discrepancy in the health profile among a sample of both lonely and non-lonely subjects ${ }^{44}$ or less physical activity and a higher tendency to smoke in lonely individuals, ${ }^{45}$ concluding in ambiguous results. However, research on social isolation produced more consequent corollary. Adults with small social networks and less social contacts, frequently adopt an unhealthy diet with elements of heavy drinking and smoking. ${ }^{46}$ Further, evidence revealed that social participation was a protective factor to frailty in older people who had a high risk of frailty over 14 years. ${ }^{47}$ The value of significant others in adjusting and preserving healthy behavioral choices through the interchange of several social cues have been recognized by most social-cognition models of health behavior. ${ }^{48}$

Grant et al in a series of experimental studies where acute stressful challenges such as color-word interference and mirror tracing tasks were utilized, found that socially isolated old participants had poorer recovery of systolic blood pressure and greater increases in total: HDL cholesterol ratio. It was, also, reported that an increased fibrinogen and natural killer cell response to stress tasks was evident in lonely participants when compared to the non-lonely ones. ${ }^{49}$ Marital status and quality of marriage seem to have a remarkable influence on blood pressure, ${ }^{50}$ with a stronger effect on older males. ${ }^{51}$ Finally, a recent systematic review in old individuals highlighted that social isolation and loneliness contribute to a poorer immune system, selective expansion of proinflammatory monocytes, enhanced expression of cytokines, and glucocorticoid resistance..$^{52}$ Mechanisms that are likely to increase the risk of coronary heart disease. ${ }^{53}$ Nonetheless, only a third of the published studies use a longitudinal design and the fact that most studies are cross-sectional means that still relatively little is known about mechanisms and causal links. ${ }^{54}$ Therefore, similar investigations should be carried out in order to obtain clearer insights on the prognosis of diverse health conditions.

\section{Interventions and future research}

Wide and systematic reviews suggest that positive outcomes and wellbeing in elderly are attainable with social engagement; ${ }^{55}$ however, the crucial question is which interventions are the most beneficial. There is a broadly accepted conviction that home visits can be effective for the bereaved and housebound adults as well as their care-givers. However, the conclusions from two systemic reviews were conflicting. Van Haastregt ${ }^{56}$ deduced that no significant fact could predict the prevention of loneliness by home-based support whereas Elkan ${ }^{57}$ concluded that home visits could prevent admission to institutional care and prolong survival. Further, a randomized control trial of a model of restorative home care on physical health and social support showed significant improvements in physical function but no changes in perceived levels of social support. ${ }^{56}$ Regarding depression, interventions counteracting social withdrawal and enabling meaningful social contact 
managed to alleviate depressive symptomatology with promising results for relapse prevention. ${ }^{58}$ Even though the descent of fundamental functions such as learning, memory and focus is widely recognized, systematic research in social gerontology and aging psychology indicates that the occupation with a more intricate activity may restrict the negative functional declines. ${ }^{59} \mathrm{~A}$ longitudinal study in a sample of US adults revealed that challenges in daily functioning such as dressing up, eating and bathing, decreased mobility and difficulty with climbing stairs over a 6-year period among lonely participants. ${ }^{60}$

Comparisons of different therapeutic approaches showed that cognitive behavioral therapy-based interventions which target maladaptive social cognition demonstrated the greatest reduction in loneliness scores. ${ }^{61}$ Also, befriending, an active practice of therapist-client communication or jointly participation in activities, showed equal improvements in both positive and negativesymptoms. ${ }^{62}$ One of these befriending programmes -focused on older women - reported success in attracting lonely older people but not in improving the well-being of participants. ${ }^{63}$ Different results were found for a club targeting men in a care home, as participants reported a significant reduction in their depression and anxiety levels. ${ }^{64}$

While research on social isolation and loneliness with their consequent mental and physical aftermaths is growing, there are many aspects still to consider. ${ }^{65}$ Several studies have confirmed the hypotheses that social isolation, loneliness, depression and other psychosocial distress may be risk factors for poor health outcomes. However, the exact mechanisms and the different types of social bonds by which social isolation and loneliness impact cardiovascular health remain elusive. Furthermore, the quantitative methodologies used to measure psychosocial distress may not adequately capture what individuals subjectively experience. ${ }^{66}$ Equally, the majority of up-to-date research has utilized samples from single countries or regions rather that expanding their search cross-nationally in both neu-

\section{References}

1. House JS. Social isolation kills, but how and why? Psychosom Med 2001, 63:273-274, doi: 10.1097/00006842-200103000-00011

2. Lauder W, Mummery K, Jones M, Caperchione C. A comparison of health behaviours in lonely and non-lonely populations. Psychol Health Med 2006, 11:233-245, doi: 10.1080/135485000500266607

3. Van Baarsen B, Snijders T, Smit JH, Van Duijn, M. Lonely but not alone: Emotional isolation and social isolation as two distinct dimensions of loneliness in older people. Educational and Psychological Measurement 2001, 61:119-135, doi: 10.1177/00131640121971103 rotypical and neurodivergent individuals. Thus, more quantitative and qualitative studies should be pursued further. Additionally, prospective studies are necessary to determine if loneliness and social networks predict the prognosis of $A D$ and to examine whether it is due to socio-structural reasons and how this relates to the increased vulnerability to health conditions. Finally, additional research is required to address whether employed interventions can have long- term positive effects and whether the potential damage that is already be done can be reversed by these interventions. The ongoing examination of social isolation and loneliness with the parallel focus on intervention evolvement is crucial for the implementation of effectual programs and for the obstruction of the vicious cycle of adverse health and psychological outcomes in elderly populations.

\section{Conclusion}

Social isolation and loneliness in older adult populations represent crucial societal phenomena that originate a sequence of multiplex brain- body interactions which ultimately makes the whole organism more susceptible to mental and physical health conditions. Thus, it is of utter importance to elucidate the causal directions that occur among social isolation, feelings of loneliness, body and brain responses. Aging is a gradual and continuous process of natural change where roles, expectations and stereotypes play a crucial role in developing personal identity and maintaining a balanced emotional state. The present review discussed and reflected on these concepts in an attempt to further elucidate the contexts, merge studies and provide attention for the amelioration of these stressful conditions. There is, still, a paucity of relevant data and a pressing need for improvement and for numerous research opportunities. Increasing knowledge base will open the path for addressing the problem with more efficient interventions and for warranting an improved quality of life for the growing population of elderly.

4. De Jong Gierveld J, Havens B. Cross-national comparisons of social isolation and loneliness: introduction and overview. Can J Aging 2004, 23:109-113, doi:10.1353/cja.2004.0021

5. Weiss R. Loneliness: The experience of emotional and social isolation. MIT Press, Cambridge, MA, 1973

6. Cornwell EY, Waite LJ. Measuring social isolation among older adults using multiple indicators from the NSHAP study. J Gerontol B Psychol Sci Soc Sci 2009b, 64:38-46, doi: 10.1093/geronb/gbp037

7. Cornwell EY, Waite LJ. Social disconnectedness, perceived isolation, and health among older adults. J Health Soc Behav 2009a, 50:31-48, doi: $10.1177 / 002214650905000103$ 
8. Boomsma DI, Cacioppo JT, Muthén B, Asparouhov T, Clark S. Longitudinal genetic analysis for loneliness in Dutch twins. Twin Res Hum Genet 2007, 10:267-273, doi10.1375/twin.10.2.267

9. Hawkley LC, Hughes ME, Waite LJ, Masi CM, Thisted RA, Cacioppo JT. From social structural factors to perceptions of relationship quality and loneliness: the Chicago health, aging, and social relations study. J Gerontol B Psychol Sci Soc Sci 2008, 63:375-384, doi:10.1093/geronb/63.6.s375

10. Savikko N, Routasalo P, Tilvis RS, Strandberg TE, Pitkälä KH. Predictors and subjective causes of loneliness in an aged population. Arch Gerontol Geriatr 2005, 41:223-233, doi:10.1016/j.archger.2005.03.002

11. Bouchard, G. How do parents react when their children leave home? An integrative review. J Adult Dev 2014, 21:69-79, doi: 10.1007/s10804013-9180-8

12. Bougea A, Despoti A, Vasilopoulos, E. Empty-nest-related psychosocial stress: Conceptual issues, future directions in economic crisis. Psychiatriki 2020, 30:329-338, doi:10.22365/jpsych.2019.304.329.

13. Charles ST. Strength and vulnerability integration: a model of emotional well-being across adulthood. Psychol Bull 2010, 136:1068-1091, doi: $10.1037 / \mathrm{a} 0021232$

14. McDonough P, Walters V. Gender and health: reassessing patterns and explanations. Soc Sci Med 2001, 52:547-559, doi: 10.1016/s0277-9536 (00)00159-3

15. Rijken A, Liefbroer A. Differences in family norms for men and women across Europe: Differences in family norms for men and women. Journal of Marriage and Family 2016, 78:1097-1113, doi: 10.1111/ jomf.12310

16. Holwerda TJ, et al. Increased risk of mortality associated with social isolation in older men: Only when feeling lonely? Results from the Amsterdam Study of the Elderly (AMSTEL). Psychol Med 2012, 42:843853, doi: 10.1073/pnas.1219686110

17. Tomaka J, Thompson S, Palacios R. The relation of social isolation, loneliness, and social support to disease outcomes among the elderly. $J$ Aging Health 2006, 18:359-384, doi:10.1177/0898264305280993

18. Fry PS, Debats DL. Self-efficacy beliefs as predictors of loneliness and psychological distress in older adults. Int J Aging Hum Dev 2002, 55:233-69, doi: 10.2190/KBVP-L2TE-2ERY-BH26.

19. Lim MH, Rodebaugh TL, Zyphur MJ, Gleeson JFM. Loneliness over time: the crucial role of social anxiety. J Abnorm Psychol 2016, 125:620630, doi: 10.1037/abn0000162

20. Cacioppo JT, Cacioppo S, Cole SW, Capitanio JP, Goossens L, Boomsma DI. Loneliness across phylogeny and a call for comparative studies and animal models. Perspect Psychol Sci 2015, 10:202-212, doi: 10.1177/1745691614564876

21. Quadt L, Esposito G, Critchley HD, Garfinkel SN. Brain-body interactions underlying the association of loneliness with mental and physical health. Neurosci Biobehav Rev 2020, 116:283-300, doi:10.1016/j. neubiorev.2020.06.015.

22. Grover S, Malhotra N. Depression in elderly: A review of Indian research. J Geriatr Ment Health 2015, 2:4-15, doi: 10.4103/2348-9995.161376

23. Cacioppo JT, Hughes ME, Waite TJ, Hawkley LC, Thisted RA. Loneliness as a specific risk factor for depressive symptoms: Cross-Sectional and longitudinal analyses. Psychol Aging 2006, 21:140-151, doi: 10.1037/ 0882-7974.21.1.140

24. Hawkley LC, Masi CM, Berry JD, Cacioppo JT. Loneliness is a unique predictor of age-related differences in systolic blood pressure. Psychol Aging 2006, 21:152-164, doi: 10.1037/0882-7974.21.1.152

25. Cacioppo JT, Hawkley LC, Thisted RA. Perceived social isolation makes me sad: 5-year cross-lagged analyses of loneliness and depressive symptomatology in the Chicago Health, Aging, and Social Relations Study. Psychol Aging 2010, 25:453-463, doi: 10.1037/a0017216

26. Erzen E, Çikrikci Ö. The effect of loneliness on depression: A meta-analysis. Int J Soc Psychiatry 2018, 64:427-435, doi: 10.1177/ 0020764018776349

27. Van der Kooy K, Van Hout H, Marwijk H, Marten H, Stenhouwer C, Beekman A. Depression and the risk for cardiovascular diseases: Systematic review and meta-analysis. Int J Geriatr Psychiatry 2007, 22:613-626, doi: 10.1002/gps.1723

28. Kop WJ, Kuhl EA, Barasch E, Jenny NS, Gottlieb SS, Gottdiener JS. Association between depressive symptoms and fibrosis markers: The Cardiovascular Health Study. Brain Behav Immun 2010, 24:229-235, doi: 10.1016/j.bbi.2009.09.017

29. Daniel K. Loneliness and Depression among University Students in Kenya? Global Journal of Human Social Science 2013, 4:11-18, doi: 10.1016/ j.jad.2014.04.070

30. Gask L, Aseem S, Waquas A, Waheed W. Isolation, feeling 'stuck' and loss of control: understanding persistence of depression in British Pakistani women. J Affect Disord 2011, 128:49-55, doi: 10.1016/j.jad. 2010.06.023

31. Wilby F. Depression and social networks in community dwelling elders: a descriptive study. Journal of Gerontological Social Work 2011, 54:246-259, doi: 10.1111/hsc.12311

32. Amieva H, Stoykova R, Matharan F, Helmer C, Antonucci TC, Dartigues JF. What aspects of social network are protective for dementia? Not the quantity but the quality of social interactions is protective up to 15 years later. Psychosom Med 2010, 72:905-911, doi: 10.1097/ PSY.0b013e3181f5e121

33. Camozzato A, Godinho C, Varela J, Kohler C, Rinaldi J, Chaves ML. The complex role of having confidant on the development of Alzheimer's disease in a community-based cohort of older people in Brazil. Neuroepidemiology 2015, 44:78-82, doi: 10.1159/000371521

34. Wilson RS, Krueger KR, Arnold SE, Schneider JA, Kelly JF, Barnes LL, et al. Loneliness and risk of alzheimer disease. Arch Gen Psychiatry 2007, 64:234-240, doi: 10.1001/archpsyc.64.2.234

35. Holwerda TJ, Deeg DJ, Beekman AT, van Tilburg TG, Stek ML, Jonker C et al. Feelings of loneliness, but not social isolation, predict dementia onset: Results from the Amsterdam Study of the Elderly (AMSTEL). $J$ Neurol NeurosurgPsychiatry 2012, 85:135-142, doi: 10.1136/jnnp-2012302755

36. Haj ME, Jardri R, Larøi F, Antoine P. Hallucinations, loneliness, and social isolation in Alzheimer's disease. Cogn Neuropsychiatry 2016, 21:1-13, doi: 10.1080/13546805.2015.1121139

37. Michael J, Park S. Anomalous bodily experiences and perceived social isolation in schizophrenia: an extension of the social deafferentation hypothesis. Schizophr Res 2016, 176:392-397, doi: 10.1016/j. schres.2016.06.013

38. Naghavi M, Orpana HM, Marczak LB, Arora M, Abbasi N, Abdulkader RS et al. Global, regional, and national burden of suicide mortality 1990 to 2016: systematic analysis for the Global Burden of Disease Study 2016. BMJ 2019, 364:194, doi: 10.1136/bmj.194

39. Li H, Xu L, Chi I. Factors related to Chinese older adults' suicidal thoughts and attempts. Aging Ment Health 2016, 20:752-761, doi: 10.1080/ 13607863.2015.1037242

40. Hawkley LC, Cacioppo JT. Loneliness and pathways to disease. Brain Behav Immun 2003, 17:98-105, doi: 10.1016/s0889-1591(02)00073-9

41. Calati R, Ferrari $C$, Marie $B$, Oasi $O$, Emilie $O$, Courteta $C P$, André $F$. Suicidal Thoughts and Behaviors and Social Isolation: A Narrative Review of the Literature. J Affect Disord 2019, 15:653-667, doi: 10.1016/j. jad.2018.11.022 
42. Chang Q, Chan, CH. Yip PS. A meta-analytic review on social relationships and suicidal ideation among older adults. Soc Sci Med 2017, 191:65-76, doi: 10.1016/j.socscimed.2017.09.003

43. Valtorta NK, Kanaan M, Gilbody S, Ronzi S, Hanratty B. Loneliness and social isolation as risk factors for coronary heart disease and stroke: systematic review and meta-analysis of longitudinal observational studies. Heart 2016, 102:1009-1016, doi: 10.1136/heartjnl-2015-308790

44. Steptoe A, Owen N, Kunz-Ebrecht SR, Brydon L. Loneliness and neuroendocrine, cardiovascular, and inflammatory stress responses in middle-aged men and women. Psychoneuroendocrinology 2004, 29:593-611, doi: 10.1016/S0306-4530(03)00086-6

45. Hawkley LC, Thisted RA, Cacioppo JT. Loneliness predicts reduced physical activity: cross-sectional \& longitudinal analyses. Health Psychol 2009, 28:354-363, doi: 10.1037/a0014400

46. Kharicha K, Iliffe S, Harari D, Swift C, Gillmann G, Stuck AE. Health risk appraisal in older people 1: Are older people living alone an "at-risk" group? Br J Gen Pract 2007, 57:271-276, doi:10.1186/1471-2296-14-130

47. Mehrabi F, Béland F. Effects of social isolation, loneliness and frailty on health outcomes and their possible mediators and moderators in community-dwelling older adults: A scoping review. Arch Gerontol Geriatr 2020, 90:104-119, doi: 10.1016/j.archger.2020.104119

48. Conner M, Norman P. Predicting health behaviour: A social cognition approach. In: M. Conner, P. Norman (eds) Predicting health behaviour: Research and practice with social cognition models. Open University Press 2005, 385:1-27, doi: 10.1016/S0925-7535(97)81483-X

49. Grant N, Hamer M, Steptoe A. Social isolation and stress-related cardiovascular, lipid, and cortisol responses. Ann Behav Med 2009, 37:29-37, doi: 10.1007/s12160-009-9081-z

50. Holt-Lunstad J, Uchino BN, Smith TW, Hicks A. On the importance of relationship quality: The impact of ambivalence in friendships on cardiovascular functioning. Ann Behav Med 2007, 33:278-290, doi: 10.1007/bf02879910

51. Blomgren J, Martikainen P, Grundy E, Koskinen S. Marital history 1971-91 and mortality 1991-2004 in England \& Wales and Finland. J Epidemiol Community Health 2010, 66:30-36, doi: 10.1136/ jech.2010.110635

52. Xia N, Li H. Loneliness, Social Isolation, and Cardiovascular Health. Antioxid Redox Signal 2018, 28:837-851, doi: 10.1089/ars.2017.7312

53. Ong AD, Uchino BN, Wethington E. Loneliness and Health in Older Adults: A Mini-Review and Synthesis. Gerontology 2016, 62:443-9, doi: 10.1159/000441651

54. Courtin E, Knapp M. Social isolation, loneliness and health in old age: a scoping review. Health Soc Care Community 2017, 25:799-812, doi: 10.1111/hsc.12311
55. Mushtaq R, Shoib S, Shah T, Mushtaq S. Relationship between loneliness, psychiatric disorders and physical health? A review on the psychological aspects of loneliness. J Clin Diagn Res 2014, 8:1-4. doi: 10.7860/JCDR/2014/10077.4828

56. Van Haastregt JC,Diederiks JPM, Van Rossum E, de Witte P, Crebolder M. Effects of preventive home visits to elderly people living in the community: systematic review. BMJ 2000, 320:754-8, doi: 10.1136/ bmj.320.7237.754

57. Elkan R, Kendrick D, Dewey M, Hewitt M, Robinson J, Blair M, Williams D, Brummell K. Effectiveness of home-based support for older people: systematic review and meta-analysis. BMJ 2001, 323:719-724, doi: 10.1136/bmj.323.7315.719

58. Parsons J, Sheridan N, Rouse P, Robinson E, Connolly M. A randomized controlled trial to determine the effect of a model of restorative home care on physical function and social support among older people. Arch Phys Med Rehabil 2013, 94: 1015-1022, doi: 10.1016/j. apmr.2013.02.003

59. Cruwys T, Haslam SA, Dingle GA, Jetten J, Hornsey MJ, Chong ED et al. Feeling connected again: interventions that increase social identification reducedepression symptoms in community and clinical settings. J Affect Disord 2014, 159:139-146, doi: 10.1016/j.jad.2014.02.019

60. Giddens A. Sociology, All Publishing House, Bucharest, 2010

61. Perissinotto CM, Stijacic Cenzer I, Covinsky KE. Loneliness in older persons: a predictor of functional decline and death. ArchIntern Med 2012, 172:1078-1083, doi: 10.1001/archinternmed.2012.1993

62. Masi CM, Chen HY, Hawkley LC, Cacioppo JT. A meta-analysis of interventions to reduce loneliness. Personal. Soc Psychol Rev 2011 15:219-266, doi: 10.1177/1088868310377394

63. Jackson H, McGorry P, Killackey E, Bendall S, Allott K, Dudgeon P et al. Acute-phase and 1-year follow-up results of a randomized controlled trial of CBT versus Befriending for first-episode psychosis: the ACE project. Psychol Med 2008, 38:725-735, doi: 10.1017/S0033291707002061

64. Martina CM, Stevens NL. Breaking the cycle of loneliness? Psychological effects of a friendship enrichment program for older women. Aging Ment Health 2006, 10:467-475, doi: 10.1080/13607860600637893

65. Gleibs IH, Haslam C, Jones JM, Alexander Haslam S, McNeill J Connolly H. No country for old men? The role of a 'Gentlemen's Club' in promoting social engagement and psychological well-being in residential care. Aging Ment Health 2011, 15:456-466, doi: 10.1080/13607863.2010.536137

66. Stoyanova SY, Giannouli V, Gergov TK. Sentimentality and nostal gia in elderly people in Bulgaria and Greece-Cross-validity of the questionnaire SNEP and cross-cultural comparison. Eur J Psychol 2017, 13:109-128, doi: 10.5964/ejop.v13i1.1202 


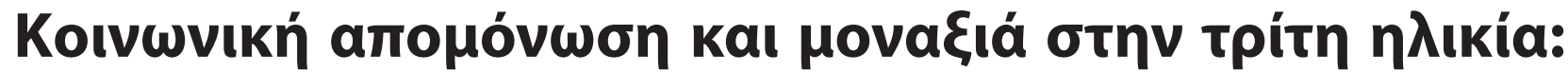

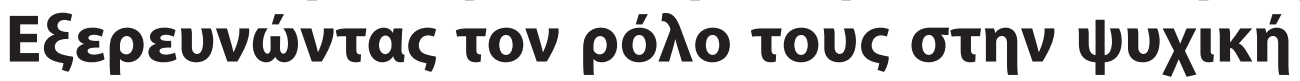

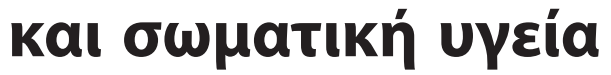

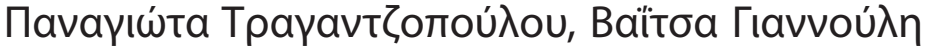

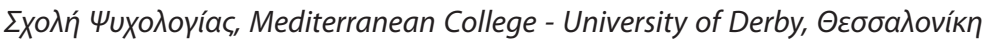

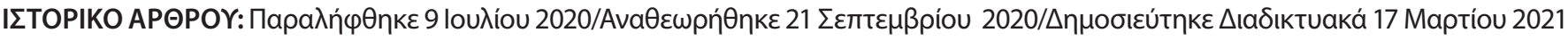

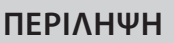

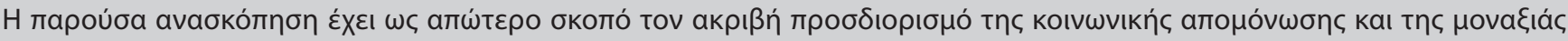

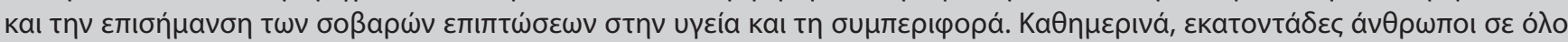

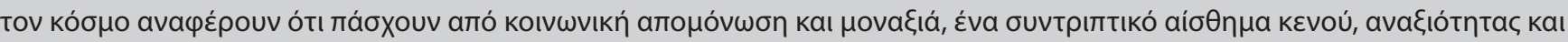

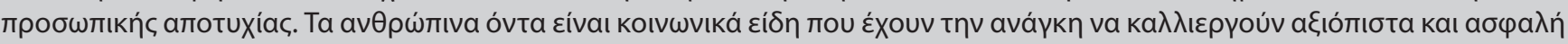

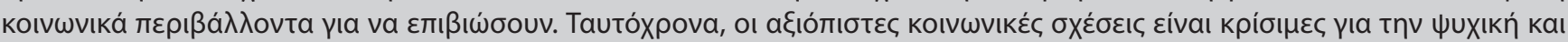

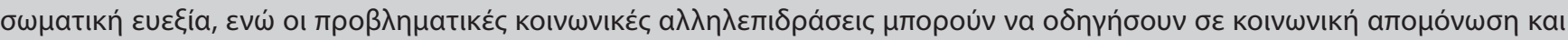

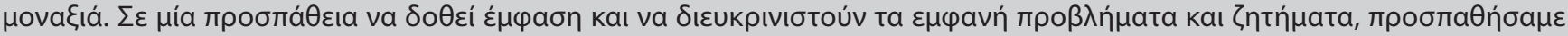

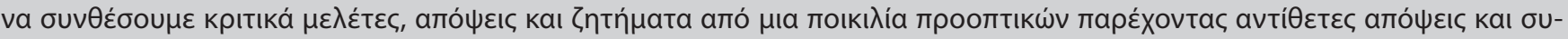

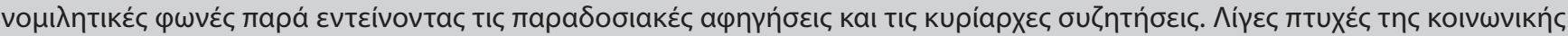

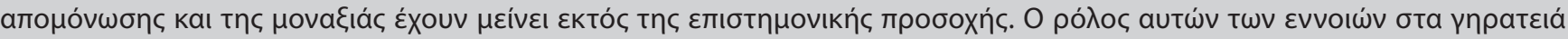

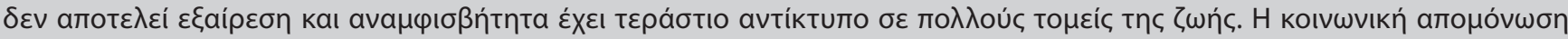

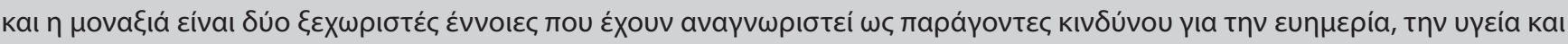

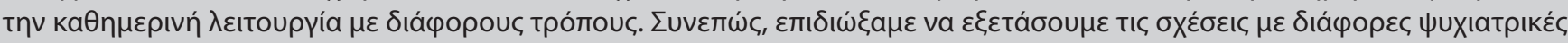

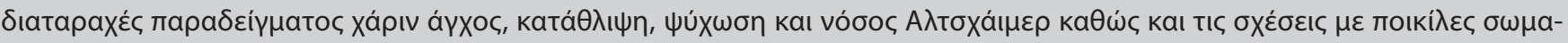

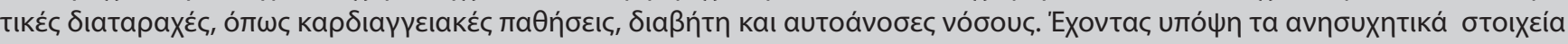

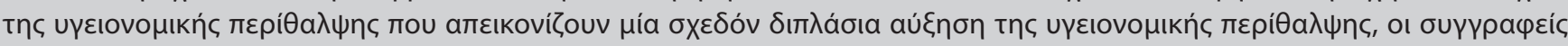

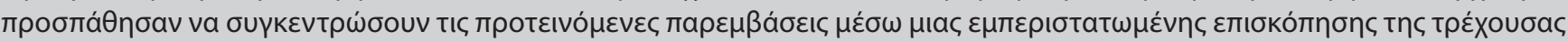

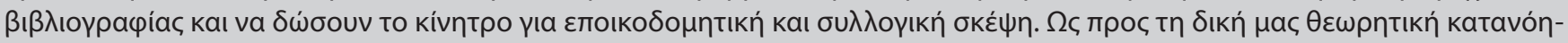

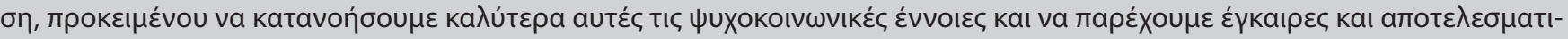

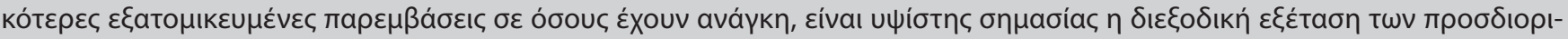

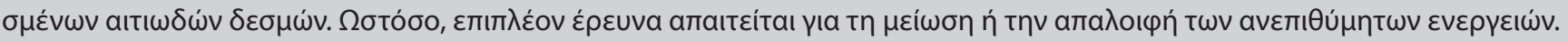

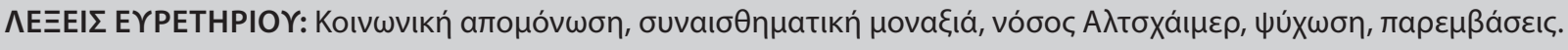

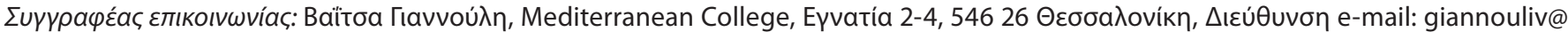
hotmail.com 